\section{$\underset{\substack{\text { hommes } \\ \text { \& migrations }}}{ }$}

\section{Hommes \& migrations}

Revue française de référence sur les dynamiques

migratoires

$1295 \mid 2012$

Algérie - France : une communauté de destin

\title{
Algérie andalouse : Lili Boniche et El Gusto
}

\section{François Bensignor}

\section{OpenEdition \\ Journals}

\section{Édition électronique}

URL : http://journals.openedition.org/hommesmigrations/1091

DOI : 10.4000/hommesmigrations. 1091

ISSN : 2262-3353

\section{Éditeur}

Musée national de l'histoire de l'immigration

\section{Édition imprimée}

Date de publication : 1 janvier 2012

Pagination : 169-175

ISSN : 1142-852X

Référence électronique

François Bensignor, «Algérie andalouse : Lili Boniche et El Gusto », Hommes \& migrations [En ligne], 1295 | 2012, mis en ligne le 29 mai 2013, consulté le 22 septembre 2020. URL : http:// journals.openedition.org/hommesmigrations/1091 ; DOI : https://doi.org/10.4000/ hommesmigrations.1091

Ce document a été généré automatiquement le 22 septembre 2020.

Tous droits réservés 


\title{
Algérie andalouse : Lili Boniche et El Gusto
}

\author{
François Bensignor
}

12012 sollicite la mémoire algérienne avec le cinquantenaire de l'accession de l'Algérie à son indépendance. Tout au long de l'année, la musique va constituer un vecteur essentiel de ce souvenir. La nostalgie d'un temps où les communautés arabes et juives enrichissaient et partageaient un patrimoine culturel commun, hérité d'al-Andalus, anime une partie de la diaspora algérienne en France. Ce phénomène, que révélait déjà il y a vingt ans le retour à la scène du regretté Lili Boniche, est confirmé par le succès remporté par le projet El Gusto.

2 Si la transmission du riche héritage musical andalou a beaucoup souffert du conflit pour la décolonisation de l'Algérie, certains maitres des styles anciens continuent d'exercer leur gusto (goût). Sous ce nom, depuis 2007, un orchestre mêlant musiciens musulmans et juifs fait revivre le chââbi de l'âge d'or. La sortie du film racontant l'aventure de sa création, à l'initiative de sa jeune réalisatrice Safinez Bousbia, inaugurait l'année, ainsi que deux concerts de l'ensemble El Gusto au Grand Rex de Paris. Le Musée d'art et d'histoire du judaïsme ouvrira à l'automne prochain l'exposition "Juifs d'Algérie", qui offrira une large place à la musique, et notamment à la mémoire du regretté Lili Boniche. Elle sera accompagnée d'un programme de films et de concerts, dans lequel El Gusto devrait figurer en bonne place. La santé déclinante de Lili Boniche ne lui a pas permis de participer à l'ensemble El Gusto, bien que sa vie ait été une magnifique illustration du propos du film. Hommage lui est rendu ici, en l'associant à l'aventure et en résumant sa carrière musicale, telle que sa fille, Karina Feredj, la raconte.

\section{Lili Boniche (1921 - 2008)}

Né dans une famille modeste, Élie, que l'on surnomme affectueusement Lili, était l'aîné de quatre enfants. Très jeune il est devenu le soutien de la famille. Son père était un artisan joaillier. Ayant perdu la vue, il ne pouvait plus exercer son métier. C'est donc au 
jeune aîné de la famille que revint la charge d'entretenir ses parents, frères et sœurs. Originaire d'Akbou en Kabylie, le père d'Élie Boniche était aussi un bon joueur de mandole. Dès l'âge de 7 ans, Lili lui chipe son instrument pour aller s'exercer sur le toit de la maison. En petit prodige et pur autodidacte, il rejoue d'oreille toutes les chansons qu'il entend chanter dans les cafés ou à la TSF. Et sa voix enfantine s'élève au-dessus des toits de la Casbah d'Alger.

3 L'immeuble qu'habite la famille Boniche se trouve au bas de la Casbah, rue Randon, une rue animée, dans laquelle vivent principalement des familles juives. Elle relie la place Rabbin-Bloch, où se dresse la grande synagogue, à la place de la Lyre avec son grand marché couvert. Dans la Casbah, on vit dehors et le petit Élie est toujours attiré par les cafés maures d'où proviennent ces musiques qui le charment. Une voix le fascine tout particulièrement, celle de Messaoud Medioni (1893-1943), dit Saoud l'Oranais. C'est un grand maître de la musique arabo-andalouse, notamment du genre haouzi, développé à Tlemcen où s'est perpétuée depuis le XVIe siècle l'école de Cordoue, et dont la transmission s'est répandue de maître à disciple jusque dans la région d'Oran.

En 1931, Lili profite d'un passage à Alger du chanteur oranais pour se présenter devant lui. Quand le jeune garçon donne de la voix, le maître Saoud, subjugué par le diamant brut qu'il vient de découvrir, décide de prendre en main sa formation en l'intégrant à son orchestre. Quelle meilleure école pourrait-il trouver? Juif, comme beaucoup de grands musiciens algériens, Saoud Medioni entend transmettre son savoir à de jeunes musiciens qui partagent la même confession. Reste à convaincre le père de Lili, qui refuse de voir son aîné s'embarquer dans une carrière de musicien... Alors qu'il se montre intraitable, Élie s'effondre en pleurs et supplications, si bien qu'il parvient à fléchir la raideur de son père. Saoud sait également trouver les mots pour obtenir son assentiment, en annonçant qu'il prend en charge tous les frais du garçon, qui recevra en outre un salaire mensuel. À dix ans, Lili rejoint ainsi l'orchestre d'un des plus célèbres chanteurs de l'époque qui lui permet de contribuera l'entretien de sa famille. $\mathrm{Au}$ sein de la troupe, il rencontre une autre jeune disciple, de six ans son aînée, Sultana Daoud, que le maitre a surnommée Reinette. Elle a perdu la vue à l'âge de 2 ans et se fera connaître sous le nom de Reinette l'Oranaise. Sur scène, Lili joue du mandole, puis du oud et s'initie surtout à la spécialité du maître : le répertoire complexe et étendu du chant oranais, hérité du haouzi. Durant trois ans, il va suivre son maître dans les galas qu'il donne à travers tout le Maghreb, sans retourner chez lui.

\section{Les débuts à la radio}

513 ans, c'est l'âge où les adolescents juifs songent à faire leur barmitsva, rite religieux marquant l'accession à l'état de personne à part entière dans la communauté. Élie demande au maître l'autorisation d'aller fêter ce moment de passage symbolique en famille à Alger. Non seulement il l'obtient, mais Saoud en personne animera la fête. Afin de compléter ses connaissances dans le domaine de la musique arabo-andalouse, Lili va alors s'initier au sein des deux plus grandes associations musicales algéroises de l'époque, El Moutribia (fondée en 1911) et El Mossilia (fondée en 1932), dont il suivra l'enseignement durant deux ans. En 1936, Lili Boniche, sans complexe et prêt à tout, décide de tenter sa chance à Radio Alger. Il rassemble quatre de ses amis avec lesquels il a l'habitude de jouer et se présente crânement au portier de la radio, son luth sous le bras. L'homme n'a pas l'intention de faire entrer ce gamin, mais se laisse fléchir par sa 
force de conviction et prévient le directeur qu'un jeune chanteur est là, qui veut passer une audition. Monsieur Azrou, qui dirige alors Radio Alger, accepte d'accorder cinq minutes au garçon, qui appelle ses amis. Les voilà en studio. Passent les cinq minutes et Lili chante ; au bout de dix minutes, il commence à s'inquiéter de l'absence de réactions du directeur ; quinze minutes s'écoulent qui lui paraissent une éternité ; enfin, au bout de vingt minutes, il voit derrière la vitre monsieur Azrou lui faire signe d'arrêter. Celuici fait irruption dans le studio et s'adresse au chanteur: "Écoute, mon petit, la semaine prochaine tu as ton émission!" Dès lors, la voix de Lili Boniche sera diffusée en direct chaque semaine dans toute l'Algérie. À 15 ans, sa carrière est lancée.

6 Ses premières prestations radiophoniques sont constituées de pièces tirées du répertoire arabo-andalou des grandes traditions oranaise et algéroise. Grâce à son émission, la réputation de Lili Boniche grandit en quelques mois. Il est bientôt sollicité de toutes parts pour animer des fêtes : mariages, baptêmes, barmitsva, etc. La radio nationale lui fait aussi bénéficier de son orchestre qui rassemble certains des meilleurs musiciens d'Algérie, comme le pianiste et chef d'orchestre Mustapha Skandrani, le violoniste Abdel Rahni ou Arlilo, joueur de derbouka réputé. À la fin des années trente, sa voix d'or est réclamée dans toute l'Algérie. Avec la guerre, les goûts du public vont évoluer. Les troupes américaines, débarquées le 8 novembre 1942, se regroupent en même temps que les forces françaises libres pour préparer l'assaut en Méditerranée. Saoud El Medioni fera partie des nombreuses victimes de la barbarie nazie. Alors qu'en 1937 il a ouvert un cabaret rue Bergère à Paris, il sera pris dans une rafle à Marseille, le 23 janvier 1943, déporté puis gazé au camp d'extermination de Sobibor. Une perte considérable pour tant de mélomanes et de disciples.

\section{Un savant mélangeur de genres}

7 En temps de guerre, on demande aux artistes de regonfler le moral des troupes. La nature enjouée de Lili Boniche l'y porte tout naturellement. Ainsi se produit-il devant les combattants de la Résistance à la demande de leurs généraux, Moraglia, chef des FFI, Pierre Weiss, etc. Au théâtre aux armées, à l'Opéra d'Alger, il chante aussi devant les soldats américains, pour lesquels il créera une chanson sur le chewing-gum... Ouvert aux nouvelles danses venues d'Amérique et des Caraïbes, Lili Boniche introduit les rythmes du tango, du paso-doble ou du mambo dans son style musical, agrémentés de paroles franco-arabes. Ces nouvelles créations intégreront son répertoire pour les fêtes. En effet, il a constaté que le public pique du nez sur les coups de minuit, après deux ou trois heures de musique classique arabo-andalouse. Avec ces chansons, qui tiennent le public éveillé jusque tard dans la nuit, le jeune chanteur donne le ton. Son nouveau style francarabe explose bientôt des deux côtés de la Méditerranée.

8 La guerre terminée, Lili Boniche est engagé au Soleil d'Algérie, un cabaret de la rue du Faubourg-Montmartre à Paris, où il se rend pour la première fois en 1946. Parmi toutes les célébrités qui fréquentent l'établissement, François Mitterrand, alors député, s'entiche des chansons du crooner algérien. Celle qu'il adore par-dessus tout, c'est L'Oriental. La joie renaît dans ce Paris de l'après-guerre. Une phrase attrapée au vol ou un bon mot suffisent à nourrir l'inspiration du chanteur. À 26 ans, Lili Boniche est porté par le tourbillon de joie qu'il contribue lui-même à créer. Jeune et beau, il plaît aux femmes. Un soir, c'est le coup de foudre! Elle se prénomme Marthe, elle est d'une élégance folle, elle porte le titre de comtesse et elle est l'épouse d'un richissime 
armateur. Un amour dévorant, exclusif, s'empare des deux amants. Mais il ne peut se satisfaire du métier du chanteur... Marthe va quitter son mari pour Élie, Élie devra quitter la chanson!

Dans les années cinquante, Lili Boniche met fin à sa première carrière musicale en France, mais continue de chanter à Alger, où il rachète quatre salles de cinéma en perte de vitesse. Il les relance grâce à son talent de programmateur et les gérera avec succès jusqu'à l'indépendance de l'Algérie. Mais il aura déjà quitté son pays natal avant le grand exode des Juifs et des pieds-noirs. Installé à Paris au début des années soixante, il acquiert d'abord un restaurant avec des amis, puis monte une société de repas pour entreprises et collectivités, Le Menu parisien, qui emploiera jusqu'à 180 personnes à l'apogée de son activité. Il pratique la musique en privé, souvent accompagné de ses anciens musiciens d'Alger avec lesquels il a gardé d'excellentes relations. Il est souvent sollicité pour chanter dans les fêtes de la communauté. Et c'est au sein de celle-ci qu'il rencontre l'âme sœur, quand son premier mariage commence à battre de l'aile. Avant le milieu des années soixante, Lili Boniche a divorcé et s'est remarié avec celle qui l'accompagnera jusqu'à la fin de ses jours.

\section{Un retour sur scène tardif et inespéré}

Sa deuxième carrière de chanteur, Lili Boniche l'entame en 1990. Il vit à cette époque une retraite tranquille et méritée, après avoir monté une entreprise de fournitures de bureau dans les années soixante-dix, puis avoir commercialisé les premières mini calculatrices de la société Commodore France. Certes, il continue à donner de petits concerts privés, mais l'opportunité qui lui est offerte d'un retour à la musique en professionnel lui apparaît comme un vrai cadeau. Son retour, il le doit à Francis Falceto. Mélomane, journaliste, homme de culture, celui-ci rêve d'entendre à nouveau sur scène la vedette du style francarabe dont il adore les disques. "Quand j'ai débarqué chez lui, je crois que c'était une grande surprise pour Lili. Ni lui, ni moi ne savions si ça allait prendre. Dès le début ça s'est bien passé dans le rapport au public (...). La greffe a pris tout de suite", explique-t-il. Parmi ses accompagnateurs, Lili Boniche retrouve le pianiste Maurice El Medioni, neveu de Saoud l'Oranais et autre retraité bientôt célébré par les professionnels et les publics internationaux des musiques du monde. Avec le violoniste Maurice Selem, ils vont tourner dans toute l'Europe et s'envoler jusqu'au Japon. Afin que l'aventure prenne sa pleine dimension, un disque reste à faire. C'est Jean Touitou, pape de la mode, qui décidera de le produire en 1996. Il porte une profonde admiration au chanteur de 75 ans installé à Cannes, et confie la direction artistique de l'album à Bill Laswell, bassiste et producteur américain réputé pour la finesse de ses goûts en matière de world music. Grâce à ces deux admirateurs, la musique de Lili Boniche pénètre les milieux les plus branchés de la toute fin du $\mathrm{XX}^{\mathrm{e}}$ siècle. Adulé des publics qui l'acclament au Barbican de Londres comme à l'Olympia de Paris ou à travers l'Europe (Allemagne, Belgique, Suède, Suisse, Italie, Espagne, etc.), le chanteur savoure ce succès formidable avec gentillesse, humour et humilité, au-delà de ses 80 ans. À chacun de ses concerts, l'émotion était au rendez-vous. Quand Safinez Bousbia contacte la fille de Lili Boniche pour lui demander s'il souhaite participer à l'aventure El Gusto, le chanteur n'est plus en mesure de monter sur scène. Bien qu'il n'ait pas pu régaler les foules au sein du grand orchestre, certaines de ses plus belles chansons figurent à son répertoire. Ainsi, son œuvre lui survit. 


\section{La naissance d'El Gusto}

11 "Ils m'ont accueilli merveilleusement. Ils m'ont entouré de leur amitié et de leur affection!" Maurice El Medioni est enthousiaste en évoquant ses retrouvailles avec les musiciens de l'ensemble El Gusto pour leur tournée de janvier 2012. Celle-ci marquait la sortie du film et du disque témoins de cette formidable aventure. Depuis le premier concert qui les a réunis en septembre 2007, le pianiste octogénaire a quitté Marseille pour s'installer à Tel Aviv auprès de ses enfants. En son fort intérieur avait germé la crainte que certains des musiciens du groupe vivant en Algérie, pays explicitement hostile à Israël, puissent affecter de l'animosité à son égard. Il n'en fut rien, bien au contraire, et son esprit qui a gardé la sensibilité de la jeunesse s'en trouve réconforté.

El Gusto réunit quelques-uns des meilleurs interprètes encore vivants de la musique chââbi algéroise, qui connut son âge d'or dans les années quarante et cinquante. Ce style issu du fonds classique andalou a nourri la culture populaire des habitants de la Casbah d'Alger, notamment à travers la poésie du malhoun marocain. Dépassé après l'indépendance de l'Algérie par le 'asri, genre constitué de chansons plus courtes et employant des instruments modernes, voilà que le chââbi renaît sous les doigts des anciens que rassemble El Gusto. L'orchestre a aussi pour vertu de renouer les fils de destins éparpillés au gré des chaos de l'histoire. Les musiciens restés dans la Casbah ont vu fermer un à un les cafés où ils se produisaient. Les occasions de toucher un cachet mariages, baptêmes, fêtes privées et autres animations de maisons closes - se faisant de plus en plus rares, ils ont fini par pratiquer cette musique pour le seul plaisir de la jouer entre eux. Quant aux musiciens juifs emportés en même temps que la vague du rapatriement des pieds-noirs en France, ils ont continué à animer les fêtes de leur communauté avec leurs airs enjoués. Mais les rares qui ont voulu poursuivre une carrière artistique ont bien dû adapter leur style à leurs nouveaux publics.

13 C'est l'intuition d'une jeune femme à l'aube de la trentaine, Safinez Bousbia, qui a tout déclenché. Née à Alger mais ayant grandi en Suisse, puis en Angleterre, en Irlande, en France et dans les Émirats arabes unis, elle découvre l'existence du chââbi pendant des vacances en 2003. Attirée par l'éclat d'un miroir dans une boutique de la Casbah d'Alger, elle est fascinée par le récit de son propriétaire, Mohamed Ferkioui, qui lui raconte son ancienne vie de musicien. Une porte s'ouvre sur un monde extraordinaire et mystérieux. Les murs lépreux de la Casbah d'aujourd'hui retrouvent leur éclat d'alors. Les patios en ruines s'animent de voix de femmes s'interpellant d'un balcon l'autre. Des mélodies de mandoles, de violons, de ouds, de derboukas et mandolines s'échappent des portes grandes ouvertes des cafés ou des salons de coiffure. Les mélismes suaves de voix de charme dévalent les ruelles... Cette vision illumine l'esprit de Safinez au point de transformer radicalement sa vie. Apprenant à aimer le joyau musical du chââbi à travers le destin chamboulé de ses interprètes, elle va concentrer toute son énergie à réinsuffler la vie à cette belle musique. Sa première mission consiste à retrouver les élèves du maître Mohamed El Anka, l'icône du chââbi, qu'il introduisit au Conservatoire d'Alger, où il fonda la première classe dans cette discipline en 1957.

14 C'est ainsi qu'elle va progressivement constituer l'orchestre. À Alger, autour d'El Hadi Halo, le fils d'El Anka, avec Rachid Berkani, Ahmed Bernaoui, Abdelkader Chercham, Mamad Haïder Benchaouch, Mohamed El Ferkioui, Abdelrahmane Guellati, Liamine Haimoune, Abdel Madjid Meskoud, Mustapha Tahmi. En France, avec les musiciens 
exilés Robert Castel, fils du grand maître Lili Labassi, Luc Cherki, Redha El Djilali, Maurice El Medioni, José de Souza, René Perez. Un seul credo pour Safinez : rassembler ces artistes séparés par les circonstances, mais toujours unis par la musique. Productrice de l'aventure, elle réussit le tour de force de mettre en scène plus de 40 musiciens lors d'une première tournée en septembre-octobre 2007, qui démarre à Marseille au Théâtre du Gymnase, passe par le Palais Omnisport de Paris Bercy, le Barbican de Londres et le JazzFest de Berlin. Une grande partie du film est réalisé au cours de cette tournée. D'autres concerts ponctuels ont lieu en 2008, 2009 et 2010. Pour la tournée de 2012, l'ensemble est constitué d'une vingtaine de musiciens et chanteurs. Plusieurs artistes de la première formation ont disparu entre- temps. Pourtant, partout cette musique fait l'effet d'un baume sur les plaies du passé. Dans les salles de concert comme dans les cinémas où l'on projette le film, les youyous fusent pour exprimer le bonheur d'être ensemble. Les jeunes ont le visage fendu de grands sourires. Et les anciens partagent cette joie qui s'abreuve de larmes nostalgiques.

\section{BIBLIOGRAPHIE}

\section{CD}

- El Gusto (Remark Records /Warner)

- Lili Boniche Anthologie (à paraître à l'automne 2012)

\section{DVD}

- El Gusto (sortie le 5 juin 2012)

- Blog El Gusto : http://elgustolefilm.wordpress.com

\section{EXPOSITION}

"Juifs d'Algérie"

Du 28 septembre 2012 au 27 janvier 2013

Musée d'art et d'histoire du judaïsme

Hôtel de Saint-Aignan

71, rue du Temple - 75003 Paris

Tél. : 0153018665 - info@mahj.org

www.mahj.org 